\title{
A Case Presentation on Rheumatic Heart Disease with Mitral Regurgitation
}

\author{
Jayasudha A. ${ }^{1} \quad$ Sreerenjini B. ${ }^{1} \quad$ Kaveri P. ${ }^{1} \quad$ Anitha P. ${ }^{1}$ \\ 1 Obstetrics and Gynaecological Nursing Department, \\ PSG College of Nursing, Coimbatore, Tamil Nadu, India
}

\begin{abstract}
Address for correspondence Anitha P, Obstetrics and Gynaecological Nursing Department, PSG College of Nursing, Coimbatore 641004, Tamil Nadu, India (e-mail: anithap920@gmail.com).
\end{abstract}

J Health Allied Sci ${ }^{\mathrm{NU}}$ 2019;9:28-30

\begin{abstract}
During any pregnancy there is an increase in blood volume of 30 to $50 \%$ resulting in increased pressure on the heart valves. For women with rheumatic heart disease this increased pressure presents increased maternal and/or fetal risks. Counseling of women with rheumatic heart disease gives appropriate surveillance of maternal and fetal well-being, as well as planning and documentation of the management of elective and emergency delivery. The care of pregnant women with rheumatic heart disease thus requires a multidisciplinary approach, involving obstetricians, cardiologists, and anesthetists. However, many women with rheumatic heart disease have healthy pregnancies and healthy babies with the right medical care.
\end{abstract}

\section{Introduction}

Rheumatic heart disease (RHD) is a disease of poverty. RHD describes a group of short-term (acute) and long-term (chronic) heart disorders that occurs as a result of acute rheumatic fever. It is usually seen in children who are 5 to 15 years old.

RHD is a disease of the young and its impact is seen in women of reproductive age. It is a preventable yet serious public health problem in low- and middle-income countries. Currently 30 million people are thought to be affected by RHD globally.

The disease results from damage to the heart valves caused by one or several episodes of rheumatic fever, an autoimmune inflammatory reaction to throat infection caused by group $\mathrm{A}$ streptococci. It is an active or inactive disease of the heart and the most affected is generally the mitral valve which is characterized by reduced functional capacity of the heart caused by inflammatory changes in the myometrium or scarring of the valves. Risk factors of RHD include poverty, poor housing, overcrowding, and malnutrition. The signs and symptoms are joint pain, fatigue, joint stiffness, tachycardia, dyspnea and weakness, a "butterfly" rash across the cheeks, sun sensitivity, and hair loss. For some of these women an initial diagnosis is made antenatal or postpartum, as they fail to tolerate the impact of the physiological changes of pregnancy on their damaged heart valves resulting in clinical decompensation.

Cardiac surgery carries significant maternal and fetal complications. Cardiac operation during pregnancy is indicated only when medical management fails. Emergency cardiac surgery during pregnancy increases fetal mortality. However, early diagnosis, appropriate management prior to pregnancy, and good functional status at the time of entering pregnancy allowed for a good maternal and neonatal outcome.

\section{Case Description}

This study describes a 26-year-old primigravida woman with $38^{5 / 7}$ weeks of gestation with known history of RHD disease with severe mitral regurgitation in the age of 14 years. She had rheumatic fever with sore throat in childhood; later she developed RHD. She received medications like tablet penicillin $G$ potassium from the onset of disease and she was on injection penicillin in 2012 for up to 18 months. She was admitted in antenatal ward with the complaints of breathlessness, palpitation, fatigue, weakness, and bilateral pitting pedal edema for 2 months' duration. On assessment, her BP was $110 / 80 \mathrm{~mm} \mathrm{Hg}$ and pulse 96 beats/min and she was conscious and oriented. She has undergone investigations like complete blood count and thyroid hormone test. Her $\mathrm{Hb}$ was $12.1 \mathrm{gm} / \mathrm{dL}$, WBC 9,400 cells, platelet 2.19 lakhs, and TSH level was $4.760 \mathrm{micro} \mathrm{IU} / \mathrm{mL}$. Then she had echo. In that the findings were severe MR with dilated left atrium and pulmonary hypertension (42 mm Hg). EF level was $63 \%$. She was on medical treatment with tablets Lasix-40 mg, Pentids-400,000 units, Eltroxin-50 $\mu \mathrm{g}$, 
Livogen, and calcium. She was on salt restriction diet with 1L fluid restriction. The mother was kept under observation.

Then she underwent the elective LSCS. She delivered an alive male neonate with the birth weight of $2.01 \mathrm{~kg}$. APGAR score was $7 / 10$ at 1 minute and 8/10 at 5 minute. Mother and baby were healthy and kept under close observation till the fourth postoperative day. Then the mother and her baby were discharged from the hospital on the seventh postoperative day with diuretics and antibiotics.

\section{Diagnosis}

History of rheumatic fever, especially if it is treated with long-term secondary prophylaxis, doesn't always lead to RHD. Physical examination is used to assess the signs of rheumatic fever, including joint pain and inflammation. Stethoscope is used to listen to the abnormal heart rhythms. Blood tests like complete blood count, culture, and ESR is done to find out any infection and detect antibodies. Chest X-ray is done to check the size of the heart and to see if there is excess fluid in the heart or lungs. Electrocardiogram is used to find out the changes seen on heart.

Echocardiography is a noninvasive ultrasound that uses sound waves to create a moving image of the heart and to measure its size and shape. The echo test may be done one or more times during pregnancy, to monitor how well the heart is performing.

\section{Management}

Preventive aspects include the following:

- Primordial prevention reduces the risk factors for rheumatic fever by covering the mouth while sneezing or coughing, proper hand washing, and maintaining distance from sick people.

- Primary prevention of rheumatic fever can be achieved through the effective treatment of streptococcal pharyngitis with penicillin.

- Secondary prophylaxis through the administration of injections of benzathine benzylpenicillin every 3 to 4 weeks to patients with a previous history of rheumatic fever and/or RHD.

\section{General Management}

Steps for general management are as follows:

- Women with moderate or severe RHD require close supervision, normally at a tertiary referral center with cardiology and intensive care facilities.

- More frequent antenatal visits are required.

- More rest is required.

- Diet is directed to restrict weight gain and prevent anemia as it increases cardiac strain.

- Infection should be avoided and properly treated.

- Hospitalization is needed if tachycardia (exceeding 100 beats/ minute) occurs.
Medical Management

Steps for medical management are as follows:

- Digoxin is indicated in atria fibrillation to slow the ventricular response and in acute heart failure to increase myocardial contractility.

- Diuretics are used in acute and chronic heart failure.

- Beta-adrenergic blockers as propranolol may be indicated for arrhythmia.

- Anticoagulants including warfarin or low molecular weight heparin should be taken to promote circulation and to prevent clotting.

- Secondary prophylaxis (antibiotics) should be administered to prevent infection.

- Oxygen supplementation is needed to improve circulation and to maintain balance between oxygen need and demand.

\section{Surgical Management}

The options for surgical management are as follows:

- Therapeutic abortion should be considered in early pregnancy.

- Cardiac surgery may be an alternative to therapeutic abortion.

\section{Effects of Pregnancy on Rheumatic Heart Disease}

During any pregnancy there is an increase in blood volume of 30 to $50 \%$ resulting in increased pressure on the heart valves. For women with RHD this increased blood pressure presents increased maternal and/or fetal risks.

This is because the added stress on the heart can result in symptomatic RHD where previously there were no symptoms.

\section{Maternal}

Pregnancy can lead to the worsening of symptoms including the following:

- Shortness of breath with simple activity.

- Waking at night out of breath.

- Pulmonary edema.

- Atria fibrillation or clotting.

These changes begin in the first trimester but peak at 28 to 30 weeks and are sustained until term, meaning most women with valvular heart disease become more symptomatic in the third trimester.

\section{Fetal}

- Abortion

- Intrauterine growth retardation

- Still birth

- Premature labor

- Asphyxia

- Respiratory distress syndrome 


\section{Nursing care}

- RHD can be very unpredictable manifesting in many atypical ways and leading to severe complications.

- Attention should be drawn to the pulse, respiration, and blood pressure during birth. Continuous monitoring of pulse and repeated blood pressure measurements should be taken.

- Fast initiation of proper treatment may be crucial for the outcome.

\section{Nursing Diagnosis}

1. Decreased cardiac output related to inadequate blood pumped by the heart to meet metabolic demands of the body as evidenced by tachycardia.

Goal: Maintaining normal cardiac output.

\section{Interventions}

- Assess heart rate, heart sounds for gallops, and blood pressure.

- Note skin color, temperature, and moisture.

- Check for peripheral pulses including capillary refill.

- Assess for reports of fatigue and reduced activity tolerance.

- Inspect fluid balance and weight gain (weigh the mother prior to breakfast).

- Monitor ECG for rate, rhythm, and ectopy.

- Provide adequate rest with semi fowler's position.

- Administer oxygen therapy as prescribed.

2. Activity intolerance related to imbalance between oxygen supply and demand as evidenced by fatigue/ weakness. Goal: Maintain normal activity.

\section{Interventions}

- Assess level of fatigue, and ability to perform ADL and other activities in relation to severity of the condition.

- Assess dyspnea on exertion, and skin color changes during rest and when active.

- Allow for rest periods between care.

- Inform of activity or exercise restrictions and to set own limits for exercise and activity.

- Inform to request assistance when needed for daily activities.

3. Ineffective family coping related to situational and developmental crises of family and child as evidenced by family expresses concern and fear about delivery process.

Goal: Mother and her relatives will be free of fear to promote coping strategies.

\section{Interventions}

- Observe for erratic behaviors (anger, tension) and perception of crisis situation.

- Assess usual family coping methods and effectiveness.

- Assess need for information and support.

- Maintain good rapport with mother and her relatives.

- Encourage mother to expression of feelings and provide factual information about delivery process.
- Clarify any misinformation and answer questions regarding disease process.

- Provide psychological support to the mother.

- Assist in identifying and using techniques to cope with and solve problems and gain control over the situation.

- Refer family for additional support and counseling, if indicated.

4. Risk for infection related to chronic illness.

Goal: To prevent infection.

\section{Interventions}

- Assess temperature, pulse, respiration, IV site, and WBC count.

- Instruct the mothers to maintain personal hygiene practices.

- Provide adequate rest and nutritional needs.

- Wash hands before giving care.

- Use sterile technique for IV maintenance.

- Administer antibiotics as ordered.

- Inform to avoid contact with infected persons.

\section{Prognosis}

Cardiovascular diseases are the leading cause of death worldwide and in all regions except Africa. In 2015, RHD was estimated to have been responsible for 3,05,000 deaths. It is also estimated that by 2030 , over 23 million people will die from cardiovascular diseases each year.

RHD may be secondary to a combination of genetic predisposition and environmental factors. Organizations such as the Indian Heart Association are working with the World Heart Federation to raise awareness about this issue.

\section{Conclusion}

RHD continues to be a major cause of cardiac disease complicating pregnancy. Women with RHD of reproductive age must receive early preconception evaluation and advice regarding the potential impact of pregnancy on their cardiovascular function. Those who chose to conceive or present after conception need management by a MDT with emphasis on identifying and avoiding triggers of decomposition and fetal anomaly/loss throughout pregnancy and the puerperium. Even though multidisciplinary management reduces the adverse events resulting in satisfactory maternal and fetal outcomes.

\section{Conflict of Interest}

None declared.

\section{References}

1 Elizabeth M. Midwifery for Nurses. 1st ed. New Delhi: CBS Publishers; 2010

2 Weiner W. High Risk Pregnancy. 4th ed. New Delhi: Elsevier Publications; 2011

3 Queenan JT, Catherine YS, Lockwood CJ. Management of High Risk Pregnancy. 1st ed. Singapore: Markono Pvt Ltd; 2012 